\title{
La asignatura "World Economic History": propuesta para una década de grados EEES bilingües
}

\author{
Julio TASCÓN FERNÁNDEZ \\ Universidad de Oviedo \\ juliotf@uniovi.es \\ Antonio José JiMÉNEZ MuÑOZ \\ Universidad de Oviedo \\ jimenezantonio@uniovi.es
}

\begin{abstract}
Resumen:
Los desafíos planteados por la adaptación al Espacio Europeo de Educación Superior (EEES) y la inmersión lingüística en inglés entre no nativos (Aprendizaje Integrado de Contenidos y Lenguas Extranjeras, AICLE) han mostrado en la asignatura World Economic History, de la Facultad de Economía y Empresa de la Universidad de Oviedo, la necesidad de introducir mejoras para asegurar que la comunicación sea exitosa y las tareas factibles. Analizamos las principales debilidades y fortalezas del contexto actual, y apuntamos reformas de la propuesta docente sostenibles durante una década, a fin de lograr mejoras para un mayor éxito de la docencia y del aprendizaje, atendiendo al detalle lingüístico sin comprometer el contenido y profundidad de la materia.
\end{abstract}

Palabras clave: Historia económica; plurilingüismo; EEES; AICLE.

\section{"World Economic History" module: a ten-year proposal for EHEA immersion degrees}

\begin{abstract}
:
The challenges of the transition to the European Higher Education Area, together with using English as a medium of instruction among non-natives, have made visible the need to make amends to the design of the World Economic History module at the Faculty of Economics and Business at the University of Oviedo, Spain. These are geared towards ensuring successful communication and task completion on the part of the lecturer and the students. Analysing the main threats and strengths of the current design and context, a series of sustainable reforms are put forward to improve the quality of teaching and learning for a decade, attending to linguistic nuances without compromising content nor its depth.
\end{abstract}

Key Words: Economic history; plurilingualism; EHEA; CLIL.

Referencia normalizada:

Tascón Fernández, J. y Jiménez Muñóz, A. (2014): La asignatura “World Economy History”: propuesta para una década de grados EEES bilingües. Historia y Comunicación Social. Vol. 19. Núm. Especial Febrero. Págs. 235-248. 
Sumario: 1. Introducción. 2. Objetivos y metodología. 3. Propuesta docente actual. 4. La comunicación en inmersión lingüística: necesidades de intervención. 5. Apoyo lingüístico. 6. Propuesta para una década: conclusiones. 7. Referencias bibliográficas.

\section{Introducción}

La adaptación al Espacio Europeo de Educación Superior (EEES) ha hecho que, desde el año 2010, muchas de las universidades ofrezcan titulaciones en una lengua que no es la oficial del país, predominando el inglés como nueva lengua de instrucción, enseñanza y aprendizaje en nuestras aulas. Esta enseñanza responde a la realidad de la libre circulación de trabajadores cualificados entre los países miembros de la Unión Europea, pero también a una mayor globalización e internacionalización, y a la competencia explícita entre las universidades de los países miembros. En los últimos años se ha dado una apresurada carrera por conseguir ofertar las titulaciones en inglés para seguir compitiendo en un mercado abierto, a menudo sin atender a cuestiones previas como la preparación del profesorado y del alumnado para esa nueva realidad. La internacionalización de nuestras universidades implica una necesidad creciente de usar una lengua vehicular común que permita la transmisión de conocimiento y la calidad de la enseñanza, sin que sea una piedra en el camino. El inglés como lengua franca dentro del mundo científico es ya una realidad, incluso en ramas humanísticas o sociales que puedan tener un fuerte apego al propio idioma. A raíz del éxito de las experiencias desarrolladas en torno al programa Erasmus de intercambio, y acrecentado por la necesidad de encontrar trabajo en el extranjero, cada vez aumenta más el número de jóvenes que, desde un primer momento, buscan iniciar sus estudios en una lengua que les permita establecer relaciones personales o laborales en un mayor número de países. La trascendencia y atractivo de los entornos de inmersión bilingüe, a día de hoy, parece poco discutible. En estos grados no sólo se pretende desarrollar el contenido sino también la lengua, y preparar al alumno para un futuro laboral globalizado. El inglés supone un valor añadido a nuestras licenciaturas, constituye una herramienta esencial en la comunicación internacional, y facilita el acceso a un puesto de trabajo en los entornos multinacionales.

Sin embargo, también son evidentes los problemas que genera la implementación de la docencia en otro idioma, particularmente si no existen los métodos de selección y formación adecuados ni se adaptan el tiempo de preparación de la docencia, ni los recursos económicos destinados a las mismas. El mayor desafío se centra en la propia docencia; en la última década, han sido muchos los estudios realizados sobre la tarea del docente y la comunicación con los alumnos en este entorno bilingüe (Butler 2005; Alonso, Grisalena y Campo, 2008; Rienties, Brouwer y Lygo-Baker, 2013), sobre las experiencias, opiniones y preocupaciones de los profesores y alumnos (Coonan, 2007; Hunt, Neofitou, \& Redford, 2009; Pavón Vázquez \& Rubio, 2010; Hunt, 2011), y las necesidades de desarrollo profesional y formación (Banegas, 2012; Hillyard, 2011; Ball y Lindsay, 2013). Por un lado, tenemos un enfoque docente y comunicativo, el Aprendizaje Integrado de Contenidos y Lenguas Extranjeras (AICLE, 
conocido en inglés como CLIL, Content and Language Integrated Learning) que se ocupa de aquellos problemas que son inherentes a enseñar en un idioma que no es el propio. Frente a esto, tenemos a un número importante de docentes que se quejan de la falta de sofisticación del inglés de sus alumnos para las competencias específicas del grado, y que recelan de poder impartir el contenido deseado al tener que atender a problemas de comunicación cuyo origen es lingüístico, cuando "no se consideran a sí mismos profesores de lengua extranjera" (Airey, 2013: 64). Como parece lógico, "pasar por alto la competencia lingüística parece poco inteligente ya que el inglés de secundaria puede ser muy diferente al inglés académico que se demanda en la universidad" (Erling y Hilgendorf, 2006: 284). Esto ha llevado a muchos a "descafeinar y simplificar el contenido para hacerlo más comprensible lingüísticamente" (Costa y Coleman, 2010: 13), lo cual ahonda en la preocupación por la calidad de la enseñanza para aquellos profesores que no son nativos de la lengua en la que enseñan (Coyle, 2008: 105-106).

Especialmente en aquellos itinerarios bilingües del área de Ciencias Sociales, dónde la palabra tiene un gran peso en la transmisión de las ideas, esta apuesta por los grados bilingües ha supuesto un reto complejo para docentes y alumnos. Las necesidad de adaptar la comunicación y sus formas, junto a los necesarios cambios metodológicos para fomentar esas capacidades hacen perentoria, sin excepción, la urgencia en mejorar la preparación lingüística previa del docente y su capacidad para fomentar la transmisión del contenido, por un lado. Por otra parte esos cambios exigen también el fomento explícito de destrezas comunicativas de las que los alumnos en su mayor parte carecen, pero que el nivel de comunicación de la asignatura exige de forma implícita. Resulta paradigmático, dentro del campo de las Humanidades, el caso que analizamos aquí, la asignatura de primer curso World Economic History en la Universidad de Oviedo. Los precursores de los actuales itinerarios bilingües en la Facultad de Economía y Empresa de la Universidad de Oviedo fueron las asignaturas bilingües que se empezaron a poner en práctica en el curso 2003-2004, hasta la impartición de un itinerario bilingüe completo (grados de Administración y Dirección de Empresas, Economía, y Contabilidad y Finanzas). La selección del profesorado comenzó en el curso 2009-10, con acreditación interna de profesores aptos para dar las asignaturas en lengua inglesa, a los que se les exigía un nivel B2 del MCER o Marco Común Europeo de Referencia para las Lenguas y superar dos cursos impartidos por profesorado especializado: "Presentaciones orales en inglés en la clase expositiva" y "Pronunciación y fonética correctiva del inglés", ambos de 30 horas. El alumnado puede escoger el itinerario bilingüe de forma voluntaria, en competición dentro de un numerus clausus, siempre que haya obtenido como mínimo una nota de 7 sobre 10 en idioma inglés en las Pruebas de Acceso a la Universidad (PAU) o, en otra modalidad de acceso, debe aportar un certificado de nivel B1 en inglés. Para el resto del alumnado existe un itinerario paralelo en el que las asignaturas se imparten en castellano. En el itinerario bilingüe, el primer año se matricularon de la asignatura que nos ocupa 36 alumnos, el segundo 48, el tercero 96 y en el curso actual 2013-2014 son de nuevo 48 alumnos, divididos en dos grupos de prácticas. Este número de alumnos es el verdadero talón de Aquiles pues estas ratios merman 
la posibilidad de un aprendizaje presencial de calidad que atienda tanto al contenido como al idioma.

\section{Objetivos y metodología}

Analizamos cómo la comunicación se ve afectada dentro de este contexto bilingüe, y cuáles son las debilidades, fortalezas, amenazas y oportunidades que presenta el cambio del idioma de impartición de esta asignatura, con el fin de dar una estabilidad al plan y a la labor docentes y ofrecer un ejemplo de programa de mejora a aquellos profesores que se hallen en la transición hacia un itinerario bilingüe. Observaremos el desglose de las principales debilidades y fortalezas del contexto actual de la asignatura, y mostraremos aquellos aspectos a perfeccionar para lograr mejoras en las que perseverar a lo largo de la próxima década. Distinguiremos primeramente entre factores externos e internos. Como factores externos, son aspectos positivos el apoyo de la propia Universidad como Campus de Excelencia Internacional (CEI, desde 2009), una dotación TIC adecuada y una amplia experiencia en el desarrollo de Convenios y acuerdos Erasmus, incluidos los Erasmus con doble titulación, además de los Suplementos al título Europeo vigentes. Por el contrario, la posible competencia de centros privados que ofrezcan enseñanzas universitarias en inglés y con bolsa de trabajo atractiva, y el alto índice de recortes presupuestarios en la Universidad son amenazas claras. Con relación a los factores internos, son fortalezas la decidida vocación internacional la Facultad, el apoyo de la Casa de las Lenguas con su oferta de cursos de inglés para el profesorado y la impartición de un curso cero preparatorio para los alumnos de grados bilingües. Destinado a que el alumnado pueda seguir con aprovechamiento las clases en lengua inglesa en cada uno de sus grados, prepara a los alumnos durante una semana para afrontar una inmersión lingüística en la que van a encontrar de entrada, como veremos, muchas dificultades. En último término, las principales debilidades estriban en el uso del inglés como medio de instrucción, al tratarse de una docencia dada por un no nativo para estudiantes no nativos y en unos presupuestos de personal netamente insuficientes para reducir la ratio alumno-profesor. Cuando en un grupo bilingüe hay más de ochenta alumnos, la evaluación y seguimiento continuos de las competencias procedimentales y lingüísticas y de los resultados del aprendizaje por parte de un profesor se hace complicada, si no imposible. Por último, en base a este análisis modificaremos la propuesta docente actual para dar cabida a una serie de mejoras que palien parcial o totalmente estas dificultades y logren maximizar aquellas ventajas de las que disponemos. 


\section{Propuesta docente actual}

El grado en ADE incluye 60 créditos de formación básica; las materias básicas son comunes para los tres grados actuales de la Facultad de Economía y Empresa y por consiguiente los alumnos que cursan la World Economic History lo hacen durante el primer semestre (septiembre-enero), desde un perfil de grado en Contabilidad y Finanzas, o bien de grado en Economía y de grado en Administración y Dirección de Empresas. La asignatura pretende realizar un análisis de la evolución de la vida económica, preferentemente de las sociedades occidentales y de sus instituciones en el proceso de crecimiento y desarrollo, a fin de diferenciar las combinaciones de los distintos modos de concretarse la preferencia social de cada sistema económico y comprender los fenómenos económicos del presente a través de la evolución económica del pasado, con referencia especial a las épocas Moderna y Contemporánea. Los 6 créditos ECTS correspondientes a la asignatura Historia Económica Mundial/ World Economic History, según la metodología y el plan docente de la asignatura alcanza un total de 150 horas entre las que requieren asistencia y aquellas que no la requieren, desglosadas como sigue (Fig.1):

\begin{tabular}{|c|c|c|c|c|c|c|c|c|c|c|}
\hline \multirow[b]{2}{*}{ Lessons } & \multirow[b]{2}{*}{ 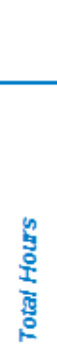 } & \multicolumn{6}{|c|}{ WORK REQUIRING ATTENDANCE } & \multicolumn{3}{|c|}{$\begin{array}{l}\text { WORK NOT REQUIRING } \\
\text { ATTENDANCE }\end{array}$} \\
\hline & & 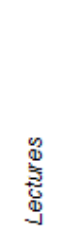 & 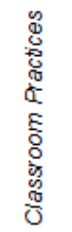 & $\begin{array}{l}\text { कू } \\
\text { है } \\
\text { है } \\
\text { कू }\end{array}$ & 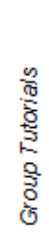 & 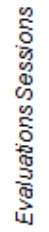 & 突 & 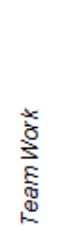 & 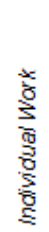 & 퓽 \\
\hline Total Hours & 150 & 22.5 & 22.5 & & 5 & 5 & 55 & 20 & 75 & 95 \\
\hline (\%) & 100 & 15 & 15 & & 3.3 & 3.3 & 36.7 & 13.3 & 50 & 63.3 \\
\hline
\end{tabular}

Fig 1. Distribución de horas de la asignatura

Tras adaptación al EESS el estudiante debe dedicar muchas más horas que antes al estudio y trabajo personal fuera del aula, a razón de 15 horas por cada 10 horas presenciales. El estudiante cuenta así con una referencia oficial que le permite atender no sólo al contenido sino también a la lengua de forma independiente, aunque siempre coordinada por el profesor. Hallamos aquí la primera necesidad de cambio metodológico. El profesor pasa a ser, por organización del tiempo, un experto pero también un facilitador de esos materiales de autoestudio, que creemos que han de ir más allá de la bibliografía recomendada en la asignatura; debe atender también a las necesidades lingüísticas y competencias comunicativas que emanen de las tareas a realizar en clase. Además, debido a la disparidad entre el nivel de inglés de entrada dentro del alumnado, resulta del todo necesario encarar ese esfuerzo por parte del alumno de manera previa a las explicaciones del profesor en el aula. De otro modo, 
tendrán bastantes dificultades para entender su discurso si, por ejemplo, desconocen el vocabulario técnico básico que se va a tratar en la clase. El docente pasa, por esa reducción de horas de contacto directo, a dar el núcleo de las explicaciones que precise cada tema y epígrafes del mismo. No se ve obligado a discutir ese tema de manera extensiva, sino adaptada al número de horas disponibles y teniendo en cuenta las horas de autoestudio, en las que además debería trabajar en su comprensión auditiva y expresión oral del idioma.

La primera modificación sobre el antiguo plan de la asignatura fue dotar al alumno de, siguiendo el Temario (Course Outline) y la Bibliografía Recomendada (Reading List), una serie de materiales que le permitan conseguir los objetivos de la asignatura. Consisten en, primeramente, una serie de textos previos a cada tema que cubran el vocabulario e ideas principales, y también orientados a las grandes temáticas que se tratarán en las Prácticas (Group Tutorials). El Previous Reading Package constituye un bloque de lecturas esenciales que deben consultar y entender previamente a las sesiones presenciales, a fin de conseguir una visión de conjunto y el vocabulario necesario. Además, el Campus Virtual alberga las presentaciones utilizadas por el profesor en clase y otros materiales relacionados, como noticias de la actualidad que motivan una reflexión sobre el pasado económico. Las clases prácticas en el aula son un complemento de las clases teóricas y deben hacer más inteligibles, si cabe, las explicaciones dadas en ellas. Asimismo, se hacen disponibles para debate posterior moderado por el profesor aquellos materiales realizados por los diferentes grupos de trabajo en las sesiones prácticas grupales, con vistas a un visión global del progreso del alumnado en el curso. El diseño de la asignatura respeta y atiende el compromiso del EEES que consiste en la utilización de metodologías más activas, el trabajo en equipo y el desarrollo de competencias generales y lingüísticas que se integren en la formación del alumno.

\section{La comunicación en inmersión lingüística: necesisdades de intervención}

El contexto de la asignatura es el de un grupo de alumnos no nativos con un profesor igualmente no-nativo, pretendiendo atender tanto a objetivos de contenido como lingüísticos. El mencionado AICLE "hace referencia a las situaciones en las que las materias o parte de las materias se enseñan a través de una lengua extranjera con un objetivo doble, el aprendizaje de contenidos y el aprendizaje simultaneo de una lengua extranjera" (Marsh, 1994: 23) y engloba una serie de principios metodológicos que analizan las necesidades creadas por la inmersión lingüística de la materia. Así, el "contenido de la materia ocurre a través de una lengua extranjera como medio de comunicación" así como "el aprendizaje de una lengua ocurre a través del contenido", de tal manera que el idioma es "una herramienta de aprendizaje y comunicación" en la que "el contenido determina el lenguaje a aprender" conforme al contexto disciplinar, lo que determina que el "léxico sea de la mayor importancia" y la enseñanza "se oriente hacia las tareas prácticas" (Marsh, 1994: 22). Como señalan los 
generadores del término, este enfoque "implica el aprender materias como la historia, la geografía u otras a través de otro idioma. Puede ser muy productivo de cara a aumentar el aprendizaje de idiomas y otras materias, y desarrollar, especialmente en alumnos jóvenes, una actitud positiva respecto a sus habilidades como aprendices y usuarios de un idioma" (Marsh, 2002: 128). El AICLE es particularmente importante en el uso del inglés como lengua franca entre dos colectivos que no son nativos en esa lengua dentro del ámbito científico. La Historia y las Ciencias Sociales, por su carácter eminentemente discursivo, han sido de las materias más adaptadas a este enfoque metodológico. La Historia de la Economía a nivel mundial pone en conexión contactos personales, referencias, textos y datos que, por su globalidad, serán en inglés aun cuando sus autores no pertenezcan al ámbito estrictamente anglosajón. La comunicación primordial será, en casi todos los casos, la misma que tenemos en clase: aquella de un no nativo con un no nativo, estableciéndose en esta asignatura no sólo una realidad virtual que prepara para el futuro, sino la propia situación a la que probablemente se enfrentará el alumno en su futuro profesional y académico. Lo que podría considerarse una debilidad, el manejo imperfecto del idioma por parte de alumnos y profesores, se convierte en una gran fortaleza ya que prepara para un contexto real de la misma manera que ejercita destrezas que son propias de la negociación de significado que se darán en el futuro ámbito laboral del alumno. El hecho de que el profesor sea un no nativo responde mejor a la realidad de cómo "el inglés encaja en nuestras sociedades multilingües" y no como "algún tipo de elemento importado" (Marsh, Baetens-Beardsmore y Hughes, 2009: 1). Los profesores no nativos pueden dar "más información sobre el idioma" y "más capaces de anticipar dificultades", y también más "empáticos con los problemas de sus alumnos", enseñar más destrezas de aprendizaje y también ser un buen ejemplo de estudiante de inglés (Reves y Medgyes, 1994: 347) para los alumnos como diestro usuario del inglés en una sociedad cada vez más global.

No obstante, la docencia en un idioma que no es el propio tiene dificultades lógicas para docentes y alumnos. Para promover la mejora en el desempeño de ambos, e incrementar el éxito de la asignatura, identificamos dos ámbitos en los que el inglés aparece como aspecto crucial a mejorar: la preparación del docente como profesor en una segunda lengua y la del alumno para entender, usar y producir en esa misma lengua. Con referencia al MCER, el nivel deseable para un profesor universitario que dé clase en un contexto de inmersión dependerá en gran medida de las tareas que tenga que realizar. No hay acuerdo en torno a cuál ha de ser el nivel de competencia lingüística de un profesor de CLIL, yendo de un "alto nivel de fluidez" (Marsh, 2002: 11) a un mero A2 para algunos cursos (Serragiotto, 2003: 62). Observando las destrezas atribuidas a cada nivel en el MCER, creemos que es bastante poco probable que la comunicación pueda ser exitosa por parte del profesor por debajo de un nivel C1 complementado por el manejo de aquellos términos específicos de la propia materia. El nivel de imprevisibilidad de la interacción en una clase con metodologías activas hace esencial que los profesores puedan responder a preguntas no preparadas, incidir sobre cuestiones que los alumnos encuentren de especial interés, o discutir textos y enfoques con un grado de espontaneidad y sin la preparación que 
posibilita el discurso continuo. Así, el nivel de inglés es un elemento esencial en el que el docente habrá de continuar trabajando a lo largo de su vida. El profesor con "mayor nivel será el mejor profesor" y por tanto "debe mejorar su uso del idioma lo antes posible" (Reves y Medgyes, 1994: 351). Los desafíos de esta asignatura y su nivel de debate e interacción hacen que ciertas dificultades terminológicas y procedimentales sean ciertamente problemáticas y excedan en ocasiones ese B2 que habilita la docencia bilingüe en esta Universidad. Asegurar el éxito de esta asignatura bilingüe en la próxima década requiere una fuerte inversión en formación por parte del profesorado, lo que casi siempre se hace de forma personal, se complica por la falta de tiempo y la fosilización de los errores de un usuario asentado en el uso del idioma. Las estancias en el extranjero o el contacto continuado con fuentes nativas de idioma pueden ayudar a paliar estos efectos (Spolsky, 1989: 118), pero tanto una como otra están comprometidas por factores económicos y sociales. Habrá que trabajar en este sentido para maximizar aquellos recursos de los que se dispone desde el entorno académico, para lo que presentamos en nuestras conclusiones una serie de acciones necesarias, factibles y sostenibles a fin de facilitar al docente universitario la mejora de sus capacidades en el otro idioma.

Sin duda, no podemos sortear el gran problema para el aprendizaje bilingüe en inmersión: los alumnos que entran en el grado bilingüe suelen adolecer de una falta de destreza para acometer las exigencias propias de estos estudios. Esa carencia, si bien es paliada en este caso por la exigencia de nota en la prueba de acceso, la práctica nos dice que no la debemos ni podemos obviar. Entre ese nivel A2 que cabe esperar a la salida del Bachillerato no se encuentra la destreza oral, que no forma parte de la evaluación de acceso a la universidad, y el peso de la comprensión oral es aún reducido frente a la gramática. Los alumnos que inician sus estudios bilingües pueden tener, por diseño educativo, grandes carencias en las destrezas lingüísticas que, en caso de no haber sido suplidas de manera no reglada, ponen en serio peligro el nivel de éxito de la asignatura. Sin que se convierta en un curso de inglés, creemos importante poder fomentar en el alumno aquellas destrezas que la experiencia en estos tres años nos dice que, como grupo, necesitan desarrollo, tales como la terminología específica, la fluidez, la pronunciación, la corrección pragmática y la capacidad para expresar y debatir ideas, a fin de dotar al alumno de herramientas que le ayuden a trabajar con los conceptos relativos a la asignatura de forma correcta. Ya se trabaja, de forma extensiva, el vocabulario pasivo en el paquete de lecturas previas, pero es necesario convertirlo en activo, así como fomentar los aspectos inherentes a la comunicación de esos conceptos de forma social, que merecen una mayor práctica y preparación por parte del alumno, siempre asistido por el docente, el cual a su vez puede apoyarse en otros expertos lingüistas que se encuentren en su Universidad. A tal fin, se han diseñado una serie de acciones y prácticas formativas que implementan los contenidos de esas lecturas en ámbitos reales y actuales a los que el alumno no es ajeno, y que podrían contribuir a su mejor comprensión de la asignatura, su aplicación y a que su aprendizaje sea más significativo. Ambas intervenciones educativas, en el ámbito del docente y del alumno, se detallan a continuación y en nuestras conclusiones. 


\section{Apoyo lingüístico}

Dejando a un lado factores externos innegociables, como son el presupuesto y el número de alumnos, y siguiendo la matriz del análisis de debilidades, amenazas, fortalezas y oportunidades y lo expuesto en la descripción del contexto AICLE, hemos identificado dos grandes factores en los que podemos incidir en nuestra acción docente, ambos enfocados en la comunicación social dentro del aula y su extensión en el futuro laboral del alumno. La principal debilidad no es otra que la del idioma vehicular, el inglés. Así, y dado que los alumnos parten de un nivel claramente insuficiente, lo que no permite siquiera seguir una clase expositiva académica (que en el MCER corresponde a niveles más altos). El nivel B2, dos niveles por encima de las capacidades de los alumnos que entran a los grados, en cuanto a recepción y producción oral los habilita para seguir una clase expositiva con éxito y poder participar de la asignatura; cualquier nivel inferior compromete seriamente esa posibilidad. Tras la experiencia de los dos primeros años de implantación del grado bilingüe, necesitamos una propuesta de asignatura en inglés mejore este aspecto sin verse comprometido el diseño de la asignatura; nótese que en algunas facultades españolas ya se ha llegado a suprimir las clases denominadas Tutorías Grupales, debido a falta de presupuesto para reformar los espacios y para contratar el número de profesores precisos para cumplir las tareas en grupos de menos alumnos. Igualmente, tampoco querríamos eliminar aquellas competencias que se refieren a la capacidad para integrarse con otros en el logro de unos objetivos comunes. Si bien el número de alumnos lo desaconseja, ya que el profesor "no podrá realizar adecuadamente la organización, observación y seguimiento de más de 6 o 7 grupos de unos 4-6 alumnos" (De Miguel, 2005: 105), el trabajo en equipo permite un desarrollo personal-social, la integración y la iniciativa, además de valores de aprendizaje inherentes como ceñirse a los objetivos e instrucciones sobre la organización y las tareas, el cronograma y la resolución de problemas dentro del grupo.

A fin de paliar las posibles deficiencias en la comunicación por parte del profesor, hemos desarrollado desde el área de Inglés para Fines Específicos del departamento de Anglogermánica y Francesa, una serie de documentos y material multimedia que sirve como elemento de reflexión acerca de la práctica de la docencia, y que a su vez ayuda a desarrollar aquellas destrezas propias de niveles avanzados $(\mathrm{C} 1 \mathrm{y} \mathrm{C} 2)$. El enfoque, como será el de la asignatura, es de CLIL aplicado a la docencia en inglés de la Historia y la Economía, y puede usarse de manera flexible como un autoaprendizaje independiente pero también como material a impartir por profesorado especializado, de forma individual o grupal (Fig. 2). Contiene los siguientes módulos:

- Tertiary education: Universities and their language (Programme of Studies, Syllabi and Module description, Admin work and registers, Lecture theatre language, Tutorial language)

- Lecturing in English (Essential Skills for CLIL, Class management, politeness and formal registers, Lecture planning and structuring, Using visuals: facts and figures, statistics and graphs, Structuring delivery: signposting, reference 
and chunking, Improving Speaking Skills: sounds and fluency, Pronunciation: intonation, rhythm and connected speech, Delivery tactics and handling questions, Assessment and marking, Problem solving)

- Teaching History and Economy (Asking questions, Comparing and contrasting, Making links through time, Cause and effect, Speaking theoretically, Interpreting sources, Applying models, Essential Maths for Economists, Money and Globalisation, Economy: yesterday, today and tomorrow)

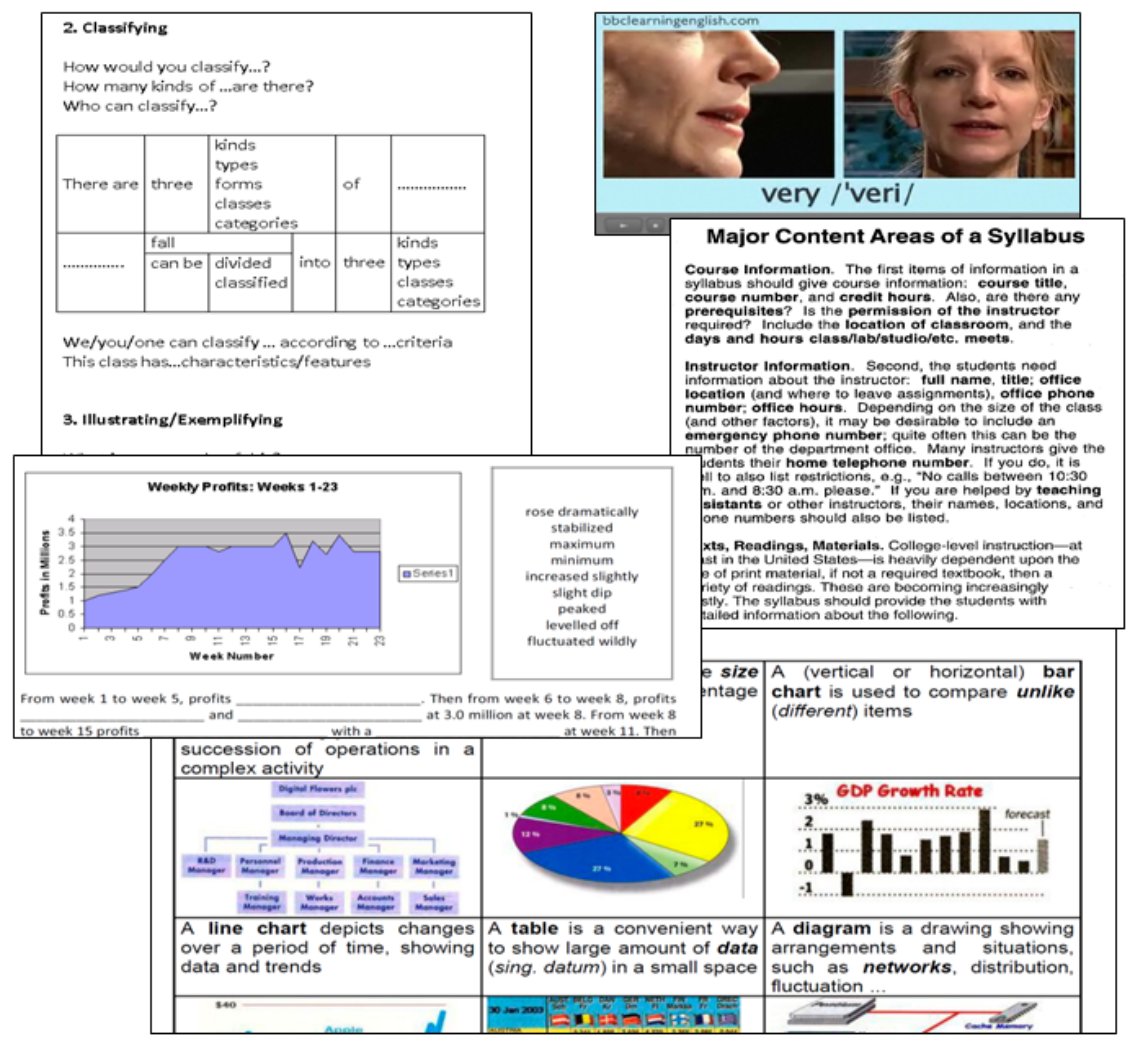

Fig 2. Ejemplo de material de autoaprendizaje

Para los alumnos, y siempre a través del Campus Virtual y de forma complementaria al curso, se han diseñado en combinación con un lingüista experto en educación una serie de materiales (Fig. 3) que el alumno tendrá a su disposición de forma permanente:

- Destrezas esenciales. Recursos orientados a desarrollar habilidades en inglés imprescindibles para la asignatura (interpretar gráficos, expresar la opinión, presentar y debatir información, escribir textos argumentativos y discursivos, 
etc.) junto a aspectos de pronunciación, lenguaje académico y acerca de la propia disciplina.

- Herramientas en línea. Acceso directo a diccionarios generales y especializados (tales como el New Palgrave Dictionary of Economics) bases de datos y sitios especializados que la ayuden a comprender y expandir sobre lo tratado, así como resolver dudas terminológicas o conceptuales.

- Palabras clave. Cada tema tiene asociados una serie de conceptos y términos esenciales que el alumno puede conocer e investigar de antemano, para los que se ofrece una serie de recursos explicativos acerca de su importancia en el contexto del curso.

- Tareas prácticas. En cada tema a tratar, se han diseñado una serie de ejercicios prácticos, pruebas y preguntas que invitan a la aplicación práctica de los aspectos vistos en clase, a la reflexión sobre la incidencia de aspectos particulares en el presente o a través de la evolución histórica. Estos se pueden abordar de forma individual, pero también en grupos, de tal manera que pueden establecerse grupos de trabajo virtual entre los alumnos.

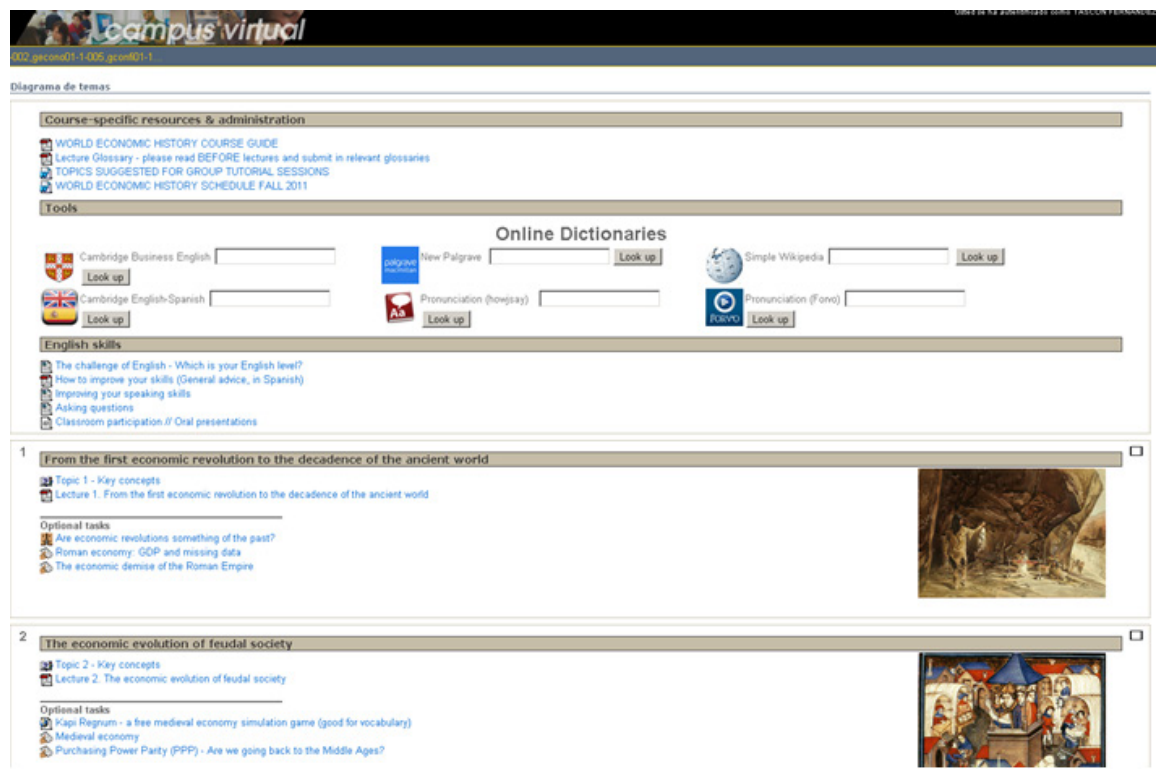

Fig 3. Vista del Campus Virtual. Detalle.

\section{Propuesta para una década: Conclusiones}

Una asignatura como Historia Económica Mundial, que arrastra tras de sí una práctica, en la universidad de Oviedo, de más de 35 cursos académicos, ha sufrido 
las correspondientes alteraciones según los vaivenes legales que emanan de las reformas educativas, incluido el cambio de asignatura anual a cuatrimestral con el plan de estudios de 1991. El paso para convertirse en World Economic History e integrarse en el EEES requiere que el modelo de la asignatura, más complejo y más frágil según se aplican estos cambios, se consolide como una oferta que mejore año tras año. Creemos que un aspecto esencial es la modularidad. Tiene un carácter semestral que legalmente señala una duración entre 12 y 14 semanas, a una media de 3 horas por semana, entre clases teóricas, prácticas de aula y tutorías grupales; por ello, nos parece prudente concretar el programa en 12 temas que aparecen divididos en 4 secciones. Igualmente, en la Reading list calificamos las lecturas de los libros referenciados como Required (obligatoria), Optional (opcional) y Highly Recomended (muy recomendada). Se intenta conseguir que el alumno combine una formación lo más sólida posible respecto a cada lección del programa, con el desarrollo de destrezas comunicativas en el idioma extranjero imbricadas con las tareas presenciales y virtuales a realizar de forma individual y grupal.

El inglés aparece como oportunidad de futuro pero amenaza de presente; con la intención de mejorar esta patente debilidad de nuestros alumnos consideramos como algo esencial la dedicación de parte de las horas de estudio a aspectos más procedimentales que faciliten la comprensión de las clases y el desempeño del alumno en esta asignatura y en otras similares en el resto del itinerario bilingüe. Hacer esto sin que parezca que estudian inglés, como algo totalmente separado de la asignatura, es un problema que hemos intentado reducir, basándonos en la propia asignatura para preparar materiales con los que el alumno pueda aprender el idioma a través de su relación con los contenidos. Todo esto se debería conseguir sin afectar el nivel de profundidad que las lecturas previas mantienen, además de transferir parte de las destrezas adquiridas al bagaje del alumno en las áreas afines. La necesidad de explicar estos aspectos a los discentes se nos antoja esencial para el éxito de este proyecto en el futuro: la cantidad de trabajo que el alumno ha de desarrollar es grande, y quizá exceda las horas de autoestudio si su nivel es bajo, pero ese esfuerzo deberá valorarse por sus potenciales beneficios en un mundo más globalizado que nunca. Desde esta óptica hemos dibujado nuestra propuesta docente para la World Economic History de los próximos diez años. Hemos intentado atender el objetivo de cómo conseguir una mejora en la preparación del profesor y de la formación del alumno en el idioma inglés sin comprometer el contenido de la asignatura, y dentro de un contexto de austeridad económica y viabilidad práctica. En otras palabras, nos hemos interrogado sobre las condiciones de la comunicación en el aula con la misma convicción con que nos interrogamos sobre el pasado económico desde la racionalidad del presente.

\section{Bibliografía}

AIREY, J. (2013). "I don't teach language: The linguistic attitudes of physics lecturers in Sweden." En: AILA Review, n 25, Amsterdam: John Benjamins. p. 64-79. 
ALONSO, E.; GRISALENA, J.; CAMPO, A. (2008). "Plurilingual education in secondary schools: Analysis of results". En: International CLIL Research Journal, nº 1(1), Jyväskylä: Universidad de Jyväskylä. p. 36-49.

BALL, P.; LINDSAY, D. (2013). "Language demands and support for English-medium instruction in tertiary education: Learning from a specific context". En DOIZ, A.; LASAGABASTER, D; SIERRA, J. M. (eds.) (2013), English-medium instruction at universities: Global challenges. Bristol, RU: Multilingual Matters. p. 44-64.

BANEGAS, D. L. (2012). "CLIL teacher development: Challenges and Experiences". En: Latin American Journal of Content \& Language Integrated Learning, $\mathrm{n}^{\circ}$ 5(1), Chía: Universidad de la Sabana. p. 46-56.

COONAN, C. M. (2007). "Insider views of the CLIL class through teacher self-observation-introspection". En: International Journal of Bilingual Education and Bilingualism, $\mathrm{n}^{\mathrm{o}}$ 10(5), Londres: Taylor and Francis. p. 625-646.

COYLE, D. (2008). "CLIL-A Pedagogical Approach from the European Perspective.” En VAN DEUSEN-SCHOLL, N.; HORNBERGER, N. (eds.) (2008), Encyclopedia of Language and Education 2nd Edition vol. 4, Nueva York: Springer. p. 1200-1214.

DE MIGUEL DÍAZ, M. (2005). Modalidades de enseñanza centradas en el desarrollo de competencias: Orientaciones para promover el cambio metodológico en el Espacio Europeo de Educación Superior. Oviedo, España: Universidad de Oviedo.

ERLING, E.; HILGENDORF, S. (2006). "Language policies in the context of German higher education." En: Language Policy, no 5, Nueva York: Springer. p. 267-292.

HILLYARD, S. (2011). "First steps in CLIL: Training the teachers." En: Latin American Journal of Content \& Language Integrated Learning, $\mathrm{n}^{\circ}$ 4(2), Chía: Universidad de la Sabana. p. 1-12.

HUNT, M. (2011). "UK teachers' and learners' experiences with of CLIL resulting in from the EU funded project ECLILT." En: Latin American Journal of Content \& Language Integrated Learning, $\mathrm{n}^{\circ}$ 4(1), Chía: Universidad de la Sabana. p. 27-39.

HUNT, M., NEOFITOU, A., \& REDFORD, J. (2009). "Developing CLIL training for modern language teacher trainees." In MARSH, D; MEHISTO, P; ALIAGA, R.; ASIKAINEN, T.; FRIGOLS, M; HUGHES, S; LANGE, G. (eds.) (2009), CLIL practice: Perspectives from the field. Jyväskylä: Universidad de Jyväskylä. p. 110-116.

MARSH, D. (1994). Bilingual Education \& Content and Language Integrated Learning. París: Universidad de la Sorbona.

MARSH, D. (2002) CLIL/EMILE, The European Dimension. Jyväskylä: Universidad de Jyväskylä.

MARSH, D.; BAETENS-BEARDSMORE, H; HUGHES, S.; MEHISTO, P.; MALJERS, A.; GRADDOL, D. (2009). "Transcript of the CLIL Debate, IATEFL 09, Cardiff", http://www.onestopenglish.com/transcript-of-the-clil-debate-iatefl09-cardiff/501068.article, [25-10-2013]. 
REVES, T.; MEDGYES, P. (1994). "The non-native English speaking EFL/ESL teacher's self-image: An international survey." En: System, no 22 (3), Waltham, MA: Elsevier. p. 353-367.

RIENTIES, B.; BROUWER, N.; LYGO-BAKER, S. (2013). "The effects of online professional development on higher education teachers' beliefs and intentions towards learning facilitation and technology." En: Teaching and Teacher Education, ${ }^{\circ}$ 29, Waltham, MA: Elsevier. p. 122-131.

SERRAGIOTTO, G. (2003). CLIL: apprendere assieme una lingua e contenuti non linguistici. Welland Ontario: Guerra.

SPOLSKY, B. (1989): Conditions for second language learning. Oxford, R.U.: Universidad de Oxford.

\section{Los autores}

Julio Tascón Fernández es Doctor en Economía por la Universidad de Oviedo. Profesor en la Facultad de Economía y Empresa, ha sido Vicedecano de Relaciones Internacionales, y profesor visitante invitado en Harvard y la London School of Economics. Es autor de, entre otros, Los Empresarios de Franco. Política y economía en España, 1936-1957 (Crítica, 2003); La inversión extranjera en España (Minerva, 2008) e Historia Económica Mundial: una visión eurocéntrica de la actividad económica, del Neolítico al siglo XXI (Biblioteca Nueva, 2012).

Antonio José Jiménez Muñoz es Doctor en Filología Inglesa por la Universidad de Oviedo. Es profesor en el área de Inglés de la Facultad de Filosofía y Letras, y de Inglés para Fines Específicos de la de Facultad de Economía y Empresa. Su principal investigación educativa, cuyos resultados publica de forma regular, analiza el éxito de la adaptación al EEES en entornos AICLE dentro del área de Economía en términos cuantitativos y cualitativos. 